\title{
Résultats nationaux de l'enquête Track auprès des utilisateurs de drogues injectables au Canada, phase 4, 2017 à 2019
}

\author{
Jill Tarasuk ${ }^{1 *}$, Jingxuan Zhang ${ }^{1}$, Anaïs Lemyre ${ }^{1}$, François Cholette², Maggie Bryson"1, \\ Dana Paquette ${ }^{1}$
}

\section{Résumé}

Contexte : L'enquête Track auprès des utilisateurs de drogues injectables (UDI) a permis de recueillir des données dans quatorze sites sentinelles au Canada (2017 à 2019).

Objectifs : Décrire la prévalence du virus de l'immunodéficience humaine $(\mathrm{VIH})$, de l'hépatite $\mathrm{C}$ et des comportements à risque associés à ceux-ci, et examiner les tendances observées au fil du temps.

Méthodes : Des informations sur les caractéristiques sociodémographiques, les déterminants sociaux de la santé, le recours aux services de prévention et au dépistage, la consommation de drogues, les comportements à risque, ainsi que le dépistage, les soins et le traitement du VIH et de l'hépatite $\mathrm{C}$ ont été recueillis par l'entremise de questionnaires administrés par un intervieweur. Les échantillons biologiques ont été analysés pour y détecter la présence d'anticorps anti-VIH et anti-hépatite $C$ et l'acide ribonucléique (ARN) de l'hépatite $C$. Les statistiques descriptives ont été calculées et les tendances observées au fil du temps ont été évaluées

Résultats : Parmi les 2383 participants, 65,6\% étaient des hommes cisgenres, 42,2 \% étaient autochtones, $48 \%$ avaient un niveau d'instruction égal ou inférieur au secondaire, $62,6 \%$ se trouvaient dans une situation de logement précaire et 75,7 \% avaient déjà été incarcérés. L'âge moyen était de 40,1 ans. La majorité des participants ont été victimes de stigmatisation et de discrimination (88,7 \%) ainsi que de violences physiques, sexuelles et/ou psychologiques durant l'enfance ( $85 \%$ ) ou de la part d'un partenaire sexuel $(75,9 \%)$. La majorité des participants ont déclaré utiliser un programme de distribution de seringues $(90,1 \%)$ et avoir été dépistés pour le VIH $(90,5 \%)$ et l'hépatite C $(90,9 \%)$.

Parmi les participants ayant des antécédents de relations sexuelles, la majorité $(59,2 \%)$ a déclaré utiliser le préservatif de manière irrégulière lors de relations vaginales ou anales avec un partenaire sexuel occasionnel. La prévalence du VIH était de 10,3\% (82,9\% d'entre eux avaient connaissance de leur statut infectieux) et de nombreux participants (36,9\%) étaient séropositifs pour l'ARN de l'hépatite C (50,1\% $d^{\prime}$ entre eux avaient connaissance de leur statut infectieux).

La plupart des indicateurs de surveillance sont restés relativement stables entre la phase 1 et la phase 4 . Des changements ont été observés au niveau des substances utilisées, et des améliorations ont été notées en ce qui concerne la prévalence du VIH et de l'hépatite $\mathrm{C}$ et les indicateurs de la cascade de soins.

Conclusion : De nombreux UDI au Canada se trouvaient dans une situation de logement précaire et étaient confrontés à des niveaux élevés de stigmatisation et de discrimination. La prévalence du VIH et de I'hépatite $C$ était élevée dans certaines régions. Ces résultats contribuent aux données probantes utilisées pour informer les mesures de prévention et de contrôle ciblées.
Cette oeuvre est mise à la disposition selon les termes de la licence internationale Creative Commons Attribution 4.0

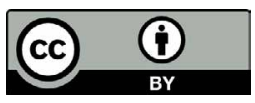

Affiliations

${ }^{1}$ Centre de la lutte contre les maladies transmissibles et les infections, Agence de la santé publique du Canada,

Ottawa, ON

2 Laboratoire national de microbiologie, Agence de la santé publique du Canada,

Winnipeg, MB

\section{${ }^{\star}$ Correspondance :}

jill.tarasuk@canada.ca

Citation proposée : Tarasuk J, Zhang J, Lemyre A, Cholette F, Bryson M, Paquette D. Résultat nationaux de l'enquête Track auprès des utilisateurs de drogues injectables au Canada, phase 4, 2017 à 2019. Relevé des maladies transmissibles au Canada 2020;46(5):155-68. https://doi.org/10.14745/ccdr.v46i05a07f

Mots-clés : $\mathrm{VIH}$, hépatite $\mathrm{C}$, utilisateurs de drogues injectables, consommation de drogues, comportements d'injection, pratiques sexuelles à risque, surdose, statut infectieux, dépistage, soins et traitement 


\section{Introduction}

Au Canada, le groupe des utilisateurs de drogues injectables (UDI) est associé à un risque important d'infection au virus de I'immunodéficience humaine $(\mathrm{VIH})$ et à l'hépatite $\mathrm{C}$. II a été estimé que, des 2165 nouveaux cas d'infection au VIH survenus en 2016, 11,3\% étaient attribuées à la consommation de drogues par injection. Ce pourcentage n'a pas diminué depuis 2014, où il était estimé à 11,2 \% (1). En Amérique du Nord, le fardeau de l'hépatite $C$ attribuée à la consommation de drogues par injection est supérieur à celui du VIH, et était estimé à 81 $\%$ en 2013 (2). Au Canada, la prévalence des anticorps anti$\mathrm{VIH}$ et anti-hépatite $\mathrm{C}$ était élevée chez les UDI interrogés de 2010 à 2012 (11,2\% et $68 \%$ respectivement) (3). Ces résultats soulignent la nécessité d'entreprendre des efforts de prévention et de traitement pour réduire les taux de morbidité et de mortalité associés au $\mathrm{VIH}$ et à I'hépatite $\mathrm{C}$ dans cette population. La surveillance biocomportementale intégrée, une approche mise en place par l'Organisation mondiale de la santé (OMS) et le Programme commun des Nations unies sur le VIH/SIDA (ONUSIDA) et adoptée à l'échelle mondiale (4), est indispensable pour informer et orienter les mesures de santé publique. Cette surveillance permet de recueillir des renseignements sur les pratiques à risque et les comportements favorisant la santé chez les populations les plus exposées au risque d'infection au $\mathrm{VIH}$.

Elle est en outre nécessaire pour mieux comprendre les facteurs contribuant à la transmission.

L'Agence de la santé publique du Canada (ASPC), en collaboration avec les provinces et les territoires, notamment les partenaires régionaux ou locaux en santé publique, surveille les tendances de la prévalence du $\mathrm{VIH}$, de l'hépatite $\mathrm{C}$ et des facteurs de risque associés chez les populations clés, comme les UDI, par l'intermédiaire du système de surveillance Track. L'enquête Track auprès des UDI (auparavant dénommée " I-Track ») consiste en la réalisation d'enquêtes transversales répétées dans certains sites sentinelles du Canada. Elle a été mise en œuvre pour la première fois de 2003 à 2005 (phase 1) dans sept sites sentinelles. Trois périodes de collecte de données ont suivi, notamment la plus récente, la phase 4 (2017 à 2019), dans quatorze sites sentinelles (annexe 1).

Ce rapport vise à présenter les résultats de surveillance nationale de la phase 4 de l'enquête Track auprès des UDI au Canada, réalisée entre le $1^{\mathrm{er}}$ janvier 2017 et le 9 mai 2019, dans les sites sentinelles canadiens participants. Ces résultats fournissent des renseignements sur les caractéristiques sociodémographiques, les déterminants sociaux de la santé, le recours aux services de prévention et au dépistage, la consommation de drogues et les antécédents de surdose, les comportements sexuels à risque ainsi que la prévalence et la cascade de soins du VIH et de l'hépatite $C$ et la connaissance du statut d'infection à ces maladies. Certains indicateurs sélectionnés au cours des phases 1 à 4 de l'enquête Track auprès des UDI sont également présentés pour décrire les tendances observées au fil du temps.

\section{Méthodes}

\section{Source de données et techniques d'échantillonnage}

Les données présentées dans ce rapport sont tirées de l'enquête Track auprès des UDI au Canada. L'enquête Track auprès des UDI se base sur l'échantillonnage par lieu de rencontre : les participants sont recrutés dans les lieux où ils ont l'habitude de se réunir, le plus souvent dans le cadre de programmes de distribution d'aiguilles et de seringues. Les personnes qui avaient consommé des drogues injectables au cours des six mois précédant le recrutement et qui avaient l'âge minimal requis pour donner leur consentement, lequel a été déterminé à chaque site selon les exigences locales en matière d'éthique de la recherche, ont été autorisées à participer à l'enquête. Les participants admissibles et consentants ont rempli un questionnaire administré par un intervieweur et fourni un échantillon biologique de sang séché (ou échantillon de salive dans le cas des sites du réseau SurvUDI).

Le protocole de surveillance et le questionnaire ont été approuvés par le Comité d'éthique de la recherche de Santé Canada et de l'Agence de la santé publique du Canada et, le cas échéant, par les comités d'éthique de la recherche de chaque site sentinelle. Les mêmes stratégies d'échantillonnage et de recrutement, ainsi que le même questionnaire de base (avec quelques révisions mineures) ont été employés lors des quatre phases pour permettre la comparabilité des résultats au fil du temps. Les méthodes d'enquête sont décrites de manière plus détaillée dans un autre document (3).

\section{Sélection des sites sentinelles}

Les sites sentinelles ont été sélectionnés suite à des consultations avec les représentants provinciaux et territoriaux, qui ont tenu compte de l'épidémiologie du VIH, de l'hépatite $\mathrm{C}$ et de la consommation de drogues et des dangers connexes. Compte tenu de ces facteurs, les sites sentinelles participants variaient selon la phase de l'enquête Track auprès des UDI (annexe 1). La collecte de données à Ottawa (Ontario) et dans la province de Québec a été coordonnée par le réseau SurvUDI (5). Les sites du réseau SurvUDI ont été répartis en quatre zones géographiques pour les analyses de la phase 4 (se reporter à l'annexe 1).

\section{Questionnaire administré par un intervieweur}

Le questionnaire de l'enquête Track auprès des UDI vise à recueillir des renseignements sur les caractéristiques sociodémographiques, les déterminants sociaux de la santé, le recours aux services de santé et de prévention (notamment le dépistage), la consommation de drogues et les comportements d'injection, les pratiques sexuelles, ainsi que les soins et le traitement du VIH et de I'hépatite $\mathrm{C}$. Le questionnaire $a$, dans un premier temps, été élaboré pour une phase pilote par un 
groupe de travail composé d'experts dans le but d'en établir la validité apparente. Pour assurer la comparabilité, la plupart des questions à l'échelle nationale ont été reprises à chaque phase ultérieure pour suivre l'évolution des informations dans le temps.

Le questionnaire de la phase 4 comporte un nombre limité de révisions, dont l'ajout de nouvelles questions à l'échelle nationale concernant l'identité de genre, les difficultés financières, l'état de santé mentale, les expériences de stigmatisation et de discrimination, les expériences de violences physiques, sexuelles et/ou psychologiques, l'emprunt de matériel usagé de consommation de drogues non injectables, les antécédents de surdose, le recours aux services de réduction des méfaits, l'absence de préservatif lors de la dernière relation sexuelle rémunérée, la consommation de substances avant ou pendant les relations sexuelles, I'observance du traitement antirétroviral et le statut de la charge virale.

\section{Échantillon biologique}

Les échantillons de sang séché ont été analysés pour y détecter la présence du VIH (anticorps et antigènes) et de I'hépatite $C$ (anticorps et ARN). Les participants n'ont pas été informés des résultats de leurs analyses en laboratoire, car aucun renseignement d'identification n'a été recueilli pour préserver l'anonymat des participants. Il a été demandé aux sites sentinelles d'assurer un service de dépistage sur place, tel l'analyse de biologie délocalisée et la phlébotomie complète, pendant les périodes de recrutement pour que les participants n'ayant pas connaissance de leur statut infectieux puissent, s'ils le souhaitaient, se faire dépister. Lorsque les sites n'étaient pas en mesure d'assurer le dépistage sur place, les participants étaient dirigés vers des centres de dépistage ou des services de soins de santé de la région. Des algorithmes actualisés d'analyse en laboratoire des échantillons de sang séché ont été introduits à la phase 4 (se reporter à l'annexe 2). Les algorithmes d'analyse des échantillons collectés dans les sites du réseau SurvUDI figurent à l'annexe 2 .

\section{Analyse}

Les statistiques descriptives des indicateurs sélectionnés ont été calculées à l'aide de SAS Enterprise Guide 7.1. Certains indicateurs sélectionnés au cours des phases 1 à 4 ont été comparés pour examiner les tendances observées au fil du temps. Les faibles valeurs numériques ont été évaluées pour déterminer le risque d'identification des participants, et laissées tel quel lorsque le risque de réidentification était jugé nul, conformément à la directive de I'ASPC sur la collecte, I'utilisation et la diffusion de l'information sur la santé publique (ASPC, 2013, document non publié). Les participants dont la réponse était "Non indiqué », " Je ne sais pas » ou "Refus » ont été exclus de chaque analyse.

\section{Résultats}

La taille des échantillons ayant participé à la phase 1, à la phase 2 et à la phase 3 était respectivement de 2 986, 2982 et 2687.

Au total, 2383 personnes étaient admissibles et ont accepté de participer à la phase 4 de l'enquête. Parmi celles-ci, 2379 (99,8 $\%)$ ont rempli un questionnaire et $2162(90,7 \%)$ ont fourni un échantillon biologique. Les résultats des indicateurs sélectionnés par caractéristiques sociodémographiques et déterminants sociaux de la santé des participants sont présentés dans les tableaux supplémentaires $A$ (indicateurs de prévention et de dépistage), B (comportements d'injection et consommation de drogues), C (comportements sexuels à risque) et $\mathbf{D}$ (indicateurs sélectionnés par phase).

\section{Caractéristiques sociodémographiques}

Dans la phase 4, 65,6\% des participants s'identifient comme hommes cisgenres, 32,7\% comme femmes cisgenres et $1 \%$ comme personnes transféminines (c'est-à-dire des personnes de sexe masculin à la naissance, mais qui s'identifient comme femmes ou non binaires) et 0,7 \% comme personnes transmasculines (c.-à-d. des personnes de sexe féminin à la naissance, mais qui s'identifient comme hommes ou non binaires) (tableau 1). L'âge moyen des participants était de 40,1 ans (tableau supplémentaire D).

Tableau 1 : Caractéristiques sociodémographiques des participants à l'enquête Track auprès des utilisateurs de drogues injectables au Canada, phase 4, 2017 à 2019 $(n=2383)$

\begin{tabular}{|c|c|c|c|c|}
\hline \multicolumn{2}{|c|}{$\begin{array}{c}\text { Caractéristiques } \\
\text { sociodémographiques }\end{array}$} & $\mathbf{n}$ & Total $^{a}$ & $\%$ \\
\hline \multirow{4}{*}{$\begin{array}{l}\text { Identité de } \\
\text { genre }\end{array}$} & Femme cisgenre & 775 & 2372 & 32,7 \\
\hline & Homme cisgenre & 1556 & 2372 & 65,6 \\
\hline & $\begin{array}{l}\text { Personne } \\
\text { transféminine }^{b}\end{array}$ & 24 & 2372 & 1,0 \\
\hline & $\begin{array}{l}\text { Personne } \\
\text { transmasculine }\end{array}$ & 17 & 2372 & 0,7 \\
\hline \multirow[t]{4}{*}{ Groupe d'âge } & Moins de 25 ans & 161 & 2378 & 6,8 \\
\hline & 25 à 39 ans & 1058 & 2378 & 44,5 \\
\hline & 40 à 54 ans & 895 & 2378 & 37,6 \\
\hline & 55 ans ou plus & 264 & 2378 & 11,1 \\
\hline \multirow[t]{7}{*}{ Site sentinelle } & Whitehorse (Yn) & 49 & 2383 & 2,1 \\
\hline & $\begin{array}{l}\text { Centre et nord } \\
\text { de l'île de } \\
\text { Vancouver (C.-B.) }\end{array}$ & 179 & 2383 & 7,5 \\
\hline & $\begin{array}{l}\text { Prince Albert } \\
\text { (Sask.) }\end{array}$ & 184 & 2383 & 7,7 \\
\hline & Regina (Sask.) & 205 & 2383 & 8,6 \\
\hline & Winnipeg (Man.) & 181 & 2383 & 7,6 \\
\hline & $\begin{array}{l}\text { Thunder Bay } \\
\text { (Ont.) }\end{array}$ & 200 & 2383 & 8,4 \\
\hline & London (Ont.) & 206 & 2383 & 8,6 \\
\hline
\end{tabular}


Tableau 1 : Caractéristiques sociodémographiques des participants à l'enquête Track auprès des utilisateurs de drogues injectables au Canada, phase 4, 2017 à 2019 ( $n=2383$ ) (suite)

\begin{tabular}{|c|c|c|c|c|}
\hline \multicolumn{2}{|c|}{$\begin{array}{c}\text { Caractéristiques } \\
\text { sociodémographiques }\end{array}$} & \multirow{2}{*}{$\begin{array}{l}\text { n } \\
157\end{array}$} & \multirow{2}{*}{$\begin{array}{r}\text { Total } \\
\\
2383\end{array}$} & \multirow{2}{*}{$\begin{array}{l}\% \\
6,6\end{array}$} \\
\hline \multirow{7}{*}{$\begin{array}{l}\text { Site sentinelle } \\
\text { (suite) }\end{array}$} & Hamilton (Ont.) & & & \\
\hline & $\begin{array}{l}\text { Ottawa (Ont.) } \\
\text { et région de } \\
\text { l'Outaouais }\left(\mathrm{Qc}_{\mathrm{c}}\right)\end{array}$ & 200 & 2383 & 8,4 \\
\hline & Montréal (Qc) & 200 & 2383 & 8,4 \\
\hline & Québec (Qc) & 125 & 2383 & 5,3 \\
\hline & $\begin{array}{l}\text { Autres sites } \\
\text { urbains au } \\
\text { Québec }^{d}\end{array}$ & 167 & 2383 & 7,0 \\
\hline & $\begin{array}{l}\text { Nouveau- } \\
\text { Brunswick }\end{array}$ & 200 & 2383 & 8,4 \\
\hline & Terre-Neuve & 130 & 2383 & 5,5 \\
\hline \multirow[t]{2}{*}{$\begin{array}{l}\text { Statut } \\
\text { d'Autochtone }\end{array}$} & $\begin{array}{l}\text { Premières } \\
\text { nations, Métis ou } \\
\text { Inuits }\end{array}$ & 997 & 2360 & 42,2 \\
\hline & Autre éthnie & 1363 & 2360 & 57,8 \\
\hline \multirow{2}{*}{$\begin{array}{l}\text { Habitant d'une } \\
\text { collectivité } \\
\text { des Premières } \\
\text { nations, des } \\
\text { Métis ou des } \\
\text { Inuits }\end{array}$} & Non & 802 & 930 & 86,2 \\
\hline & Oui & 128 & 930 & 13,8 \\
\hline
\end{tabular}

Abréviations : C.-B., Colombie-Britannique; Man., Manitoba; Ont., Ontario; Qc, Québec; Sask., Saskatchewan; Yn, Yukon

a Le total représente le nombre total de personnes associées à l'indicateur concerné, à l'exception des personnes ayant répondu par "Je ne sais pas ", "Refus " et "Non indiqué "

Les personnes transféminines inclut les personnes de sexe masculin à la naissance, mais qui s'identifient comme femmes ou non binaires

c Les personnes transmasculines inclut les personnes de sexe féminin à la naissance, mais qui s'identifient comme hommes ou non binaires

${ }^{d}$ Les autres sites urbains de la province de Québec étaient l'Abitibi-Témiscamingue, la

Montérégie, le Saguenay-Lac-Saint-Jean, les Cantons de l'Est et la Mauricie-Centre-du-Québec

- Cette question a seulement été posée aux participants autochtones

Sur l'ensemble des participants, 42,2 \% s'identifiaient comme autochtones, dont $82,9 \%$ comme membres des Premières nations, $14,9 \%$ comme membres des Métis et 2,2 \% comme membres des Inuits. Parmi les participants autochtones, 13,8 \% ont déclaré vivre dans une collectivité des Premières nations, des Métis ou des Inuits au moment de l'entrevue. La proportion de participants qui s'identifiaient comme étant d'une autre ethnie était de $57,8 \%$ et, parmi ceux-ci, la majorité $(96,3 \%$ ) s'identifiait comme Blancs. La plupart des données démographiques sont restées relativement stables au cours des quatre phases, tandis que l'âge moyen a légèrement augmenté, de même que la proportion de personnes qui s'identifiaient comme autochtones (tableau supplémentaire D).

\section{Déterminants sociaux de la santé}

Parmi les participants de la phase 4, un peu moins de la moitié (48\%) avaient un niveau d'instruction égal ou inférieur inférieur au secondaire et une large proportion (86 \%) de participants avaient rencontré des difficultés financières (mal à « joindre les deux bouts ») au cours des douze mois précédant l'entrevue (tableau 2). Globalement, $62,6 \%$ des participants se trouvaient
Tableau 2 : Déterminants sociaux de la santé des participants à l'enquête Track auprès des utilisateurs de drogues injectables au Canada, phase 4, 2017 à 2019

$(n=2383)$

\begin{tabular}{|c|c|c|c|c|}
\hline \multicolumn{2}{|c|}{$\begin{array}{l}\text { Déterminants sociaux de la } \\
\text { santé }\end{array}$} & $\mathrm{n}$ & Total $^{\mathrm{a}}$ & $\%$ \\
\hline \multirow{3}{*}{$\begin{array}{l}\text { Niveau } \\
\text { d'instruction le } \\
\text { plus élevé }\end{array}$} & $\begin{array}{l}\text { Études } \\
\text { secondaires } \\
\text { non } \\
\text { terminées }\end{array}$ & 1139 & 2373 & 48,0 \\
\hline & $\begin{array}{l}\text { Études } \\
\text { secondaires } \\
\text { terminées }\end{array}$ & 621 & 2373 & 26,2 \\
\hline & $\begin{array}{l}\text { Études } \\
\text { supérieures } \\
\text { réalisées } \\
\text { après les } \\
\text { études } \\
\text { secondaires }\end{array}$ & 613 & 2373 & 25,8 \\
\hline \multirow{2}{*}{$\begin{array}{l}\text { Difficultés } \\
\text { financières }^{\mathrm{b}} \text { au } \\
\text { cours des douze } \\
\text { derniers mois }\end{array}$} & Non & 207 & 1479 & 14,0 \\
\hline & Oui & 1272 & 1479 & 86,0 \\
\hline \multirow{2}{*}{$\begin{array}{l}\text { Situation de } \\
\text { logement } \\
\text { précaire au } \\
\text { cours des six } \\
\text { derniers mois }\end{array}$} & $\begin{array}{l}\text { Situation de } \\
\text { logement } \\
\text { précaire }^{c}\end{array}$ & 1486 & 2374 & 62,6 \\
\hline & $\begin{array}{l}\text { Situation de } \\
\text { logement } \\
\text { stable }\end{array}$ & 888 & 2374 & 37,4 \\
\hline \multirow{2}{*}{$\begin{array}{l}\text { Antécédents } \\
\text { d'incarcération }^{d}\end{array}$} & Non & 422 & 1736 & 24,3 \\
\hline & Oui & 1314 & 1736 & 75,7 \\
\hline \multirow{2}{*}{ Santé mentale } & $\begin{array}{l}\text { Passable à } \\
\text { excellente }\end{array}$ & 1401 & 1668 & 84,0 \\
\hline & Mauvaise & 267 & 1668 & 16,0 \\
\hline \multirow{2}{*}{$\begin{array}{l}\text { Antécédents de } \\
\text { stigmatisation } \\
\text { et de } \\
\text { discrimination }\end{array}$} & Non & 166 & 1464 & 11,3 \\
\hline & Oui & 1298 & 1464 & 88,7 \\
\hline \multirow{2}{*}{$\begin{array}{l}\text { Antécédents } \\
\text { de violences } \\
\text { physiques, } \\
\text { sexuelles et/ou } \\
\text { psychologiques } \\
\text { durant l'enfance }\end{array}$} & Non & 220 & 1463 & 15,0 \\
\hline & Oui & 1243 & 1463 & 85,0 \\
\hline \multirow[b]{2}{*}{$\begin{array}{l}\text { Antécédents } \\
\text { de violences } \\
\text { physiques, } \\
\text { sexuelles et/ou } \\
\text { psychologiques } \\
\text { de la part d'un } \\
\text { partenaire } \\
\text { sexuel }\end{array}$} & Non & 351 & 1458 & 24,1 \\
\hline & Oui & 1107 & 1458 & 75,9 \\
\hline \multicolumn{5}{|c|}{ 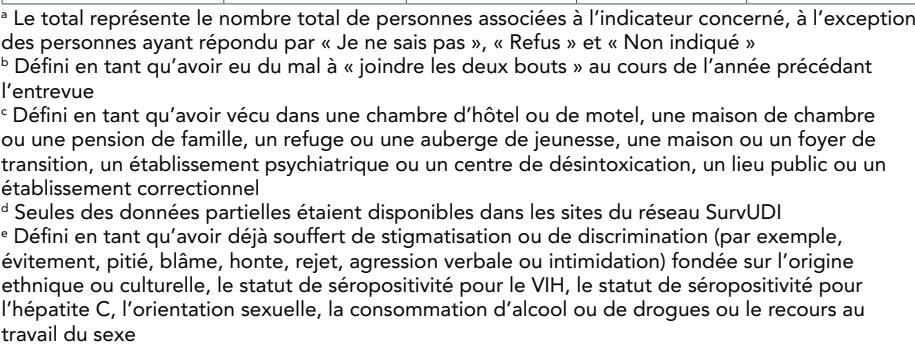 } \\
\hline
\end{tabular}


dans une situation de logement précaire au cours des six mois précédant l'entrevue, et 75,5\% ont déclaré avoir déjà été incarcérés. Une large proportion de participants (84\%) ont déclaré que leur santé mentale était " passable à excellente » et $16 \%$ ont déclaré qu'elle était « mauvaise ». Parmi les participants autochtones, $83,1 \%$ avaient fréquenté un pensionnat autochtone ou comptaient dans leur famille une personne qui avait fréquenté un pensionnat autochtone.

La majorité des participants (88,7\%) ont déclaré avoir souffert de stigmatisation et de discrimination (liée à l'origine ethnique ou culturelle, au statut de séropositivité pour le VIH, au statut de séropositivité pour l'hépatite $C$, à l'orientation sexuelle, à la consommation d'alcool ou de drogues ou au travail du sexe). Une large proportion de participants ont souffert de violences physiques, sexuelles et/ou psychologiques durant l'enfance (85\%) ou de la part d'un partenaire sexuel $(75,9 \%)$.

Au cours des quatre dernières phases, les indicateurs des déterminants sociaux sont restés relativement stables, à l'exception d'une hausse de la proportion de participants qui se trouvaient dans une situation de logement précaire au cours des six mois précédant l'entrevue (51,1\% à 62,6\%) (tableau supplémentaire D)

\section{Recours aux services de prévention et au dépistage}

Au cours de la phase 4 , la majorité des participants $(90,1 \%)$ a déclaré avoir eu recours à un programme de distribution d'aiguilles et de seringues au cours des douze mois précédant I'entrevue. Une plus faible proportion a déclaré avoir eu recours à la méthadone, à la buprénorphine ou à un autre traitement de substitution aux opiacés (47,3\%) et avoir fréquenté un site d'injection ou de consommation supervisée (13,5\%). La majorité des participants ont déclaré avoir passé des tests de dépistage pour le VIH $(90,5 \%)$ et de l'hépatite C $(90,9 \%)$ (tableau 3). Certains participants (14,3\%) avaient entendu parler de la prophylaxie préexposition (PrEP). Parmi les participants qui n'ont pas déclaré de diagnostic d'infection au $\mathrm{VIH}, 0,3 \%$ avaient utilisé la PrEP au cours des douze mois précédant l'entrevue pour réduire le risque de contracter le $\mathrm{VIH}$. La proportion de participants ayant passé des tests de dépistage pour le VIH (90 \% à 92,9 \%) et de l'hépatite C (87,5 \% à 91,3\%) était élevée et variait peu sur l'ensemble des phases (tableau supplémentaire D).

\section{Comportements d'injection}

Au cours de la phase 4 , plus d'un tiers des participants $(38,1 \%)$ ont déclaré s'être injectés quotidiennement au cours du mois précédant l'entrevue, et plus de la moitié $(52,7 \%)$ ont déclaré s'être injectés dans un lieu public au cours des six mois précédant l'entrevue. Globalement, 11,6 \% des participants se sont injectés avec des aiguilles ou des seringues usagées au cours des six mois précédant l'entrevue et, parmi eux, une
Tableau 3 : Recours aux services de prévention et au dépistage du VIH et de l'hépatite $C$ par des participants à l'enquête Track auprès des utilisateurs de drogues injectables au Canada, phase 4, 2017 à $2019(n=2$ 383)

\begin{tabular}{|c|c|c|c|}
\hline $\begin{array}{c}\text { Recours aux services de prévention et au } \\
\text { dépistage }\end{array}$ & $n$ & Total ${ }^{\mathrm{a}}$ & $\%$ \\
\hline $\begin{array}{l}\text { Recours à un programme de distribution } \\
d^{\prime} \text { aiguilles et de seringues au cours des douze } \\
\text { derniers mois }^{b}\end{array}$ & 1490 & 1653 & 90,1 \\
\hline $\begin{array}{l}\text { Fréquentation d'un site d'injection ou de } \\
\text { consommation supervisée au cours des douze } \\
\text { derniers mois }^{\text {b }}\end{array}$ & 223 & 1652 & 13,5 \\
\hline $\begin{array}{l}\text { Recours à la méthadone, à la buprénorphine ou } \\
\text { à un autre traitement de substitution aux opiacés } \\
\text { au cours des douze derniers mois }\end{array}$ & 780 & 1650 & 47,3 \\
\hline Antécédents de dépistage du VIH & 2080 & 2299 & 90,5 \\
\hline Antécédents de dépistage du VHC & 2086 & 2296 & 90,9 \\
\hline
\end{tabular}

majorité de participants (85,2\%) empruntaient des aiguilles ou des seringues à des personnes qu'ils connaissaient bien (par exemple, membres de la famille, amis ou partenaires sexuels). Plus d'un tiers des participants (38\%) se sont injectés avec du matériel d'injection usagé ( $p$. ex. eau, filtres, réchauds, garrots, ouate, acidifiants) au cours des six mois précédant l'entrevue. Parmi les participants qui empruntaient du matériel usagé, la majorité d'entre eux $(82,9 \%)$ ont déclaré l'avoir emprunté auprès de personnes qu'ils connaissaient bien. Plus de la moitié des participants (56\%) avaient emprunté du matériel de consommation usagé autre que du matériel d'injection tel que des pailles, des billets de banque ou des pipes au cours des six mois précédant l'entrevue (tableau 4).

La proportion de participants ayant déclaré avoir emprunté des aiguilles et/ou des seringues usagées a diminué de près de la moitié, passant de $20,2 \%$ lors de la phase 1 et $21,8 \%$ lors de la phase 2 à 11,6\% lors de la phase 4 . En revanche, la proportion de participants ayant déclaré avoir emprunté d'autres matériels $d$ 'injection usagés (p. ex. eau, filtres, réchauds, cuillères, garrots, attaches, ouate et acidifiants) a augmenté de près d'un tiers, passant de $29,8 \%$ lors de la phase 1 à $38 \%$ lors de la phase 4 (tableau supplémentaire D).

\section{Consommation de drogues et antécédents de surdose}

Au cours de la phase 4, la cocaïne était la drogue la plus fréquemment injectée au cours des six mois précédant I'entrevue (60\%), suivie de I'hydromorphone $(50,1 \%)$, de la méthamphétamine $(43,5 \%)$, de la morphine $(41,6 \%)$ et de I'héroïne (32,4\%). Les participants consommaient un large éventail de drogues non injectables au cours de la même période, le plus souvent du cannabis $(72,1 \%)$, de l'alcool $(62,5 \%)$, du crack $(47,8 \%)$, de la cocaïne $(46,6 \%)$ et de la 
méthamphétamine (43\%). Les participants ont également déclaré consommer des analgésiques opioïdes, par voies non injectables, en particulier la méthadone (35\%), l'hydromorphone $(28,2 \%)$, la codéine $(27,5 \%)$, la morphine $(24,7 \%)$, le fentanyl $(19,8 \%)$, l'héroïne (19,7 \%) et l'oxycodone (15,6\%) (tableau 5).

Tableau 4 : Comportements d'injection des participants à l'enquête Track auprès des utilisateurs de drogues injectables au Canada, phase 4, 2017 à 2019 ( $n=2$ 383)

\begin{tabular}{|c|c|c|c|}
\hline Comportements d'injection & $\mathbf{n}$ & Total $^{a}$ & $\%$ \\
\hline $\begin{array}{l}\text { Pratique quotidienne de l'injection au cours } \\
\text { du dernier mois }{ }^{b}\end{array}$ & 822 & 2155 & 38,1 \\
\hline $\begin{array}{l}\text { Consommation de drogues injectables dans } \\
\text { un lieu public au cours des six derniers mois }\end{array}$ & 1243 & 2357 & 52,7 \\
\hline $\begin{array}{l}\text { Emprunt d'aiguilles ou de seringues } \\
\text { usagées au cours des six derniers mois }\end{array}$ & 271 & 2339 & 11,6 \\
\hline $\begin{array}{l}\text { Emprunt d'aiguilles ou de seringues } \\
\text { usagées auprès de personnes bien connues } \\
\text { au cours des six derniers mois }\end{array}$ & 224 & 263 & 85,2 \\
\hline $\begin{array}{l}\text { Emprunt d'autres matériels d'injection } \\
\text { usagés (p. ex. eau, filtres, réchauds, garrots, } \\
\text { ouate, acidifiants) au cours des six derniers } \\
\text { mois }\end{array}$ & 882 & 2324 & 38,0 \\
\hline $\begin{array}{l}\text { Emprunt d'autres matériels d'injection } \\
\text { usagés auprès de personnes peu connues } \\
\text { au cours des six derniers mois }\end{array}$ & 710 & 856 & 82,9 \\
\hline $\begin{array}{l}\text { Emprunt de matériel de consommation } \\
\text { usagé autre que du matériel d'injection } \\
\text { (p. ex. des pailles, des billets de banque ou } \\
\text { des pipes) au cours des six derniers mois }{ }^{b}\end{array}$ & 1153 & 2059 & 56,0 \\
\hline
\end{tabular}

a Le total représente le nombre total de personnes associées à l'indicateur concerné, à l'exception des personnes ayant répondu par "Je ne sais pas ", "Refus » et " Non indiqué "

Cette question n'a pas été posée au site de London

"Les "personnes bien connues " est défini en tant que des membres de la famille, des amis ou des partenaires sexuels

Tableau 5 : Consommation de drogues et antécédents de surdose des participants à l'enquête Track auprès des utilisateurs de drogues injectables au Canada, phase 4, 2017 à $2019(n=2383)$

\section{Consommation de drogues et} antécédents de surdose

n $\quad$ Total $^{\mathrm{a}} \quad \%$

Types de drogues utilisées par injection au cours des six derniers mois ${ }^{b}$

\begin{tabular}{|l|r|r|r|}
\hline Cocaïne & 1419 & 2364 & 60,0 \\
\hline Hydromorphone & 1184 & 2363 & 50,1 \\
\hline Méthamphétamine & 1027 & 2360 & 43,5 \\
\hline Morphine & 982 & 2362 & 41,6 \\
\hline Héroïne & 764 & 2357 & 32,4 \\
\hline Fentanyl & 572 & 2350 & 24,3 \\
\hline Amphétamines & 506 & 2358 & 21,5 \\
\hline Crack & 473 & 2362 & 20,0 \\
\hline Ritalin (seul) & 466 & 2361 & 19,7 \\
\hline Oxycodone & 400 & 2365 & 16,9 \\
\hline Hérö̈ne et cocaïne & 330 & 2359 & 14,0 \\
\hline Benzodiazépines & 173 & 2361 & 7,3 \\
\hline Talwin et Ritalin & 166 & 2359 & 7,0 \\
\hline Méthadone & 145 & 2366 & 6,1 \\
\hline
\end{tabular}

Tableau 5 : Consommation de drogues et antécédents de surdose des participants à l'enquête Track auprès des utilisateurs de drogues injectables au Canada, phase 4, 2017 à $2019(n=2383)$ (suite)

\section{Consommation de drogues et} antécédents de surdose

n $\quad$ Total ${ }^{\mathrm{a}}$

$\%$

Types de drogues utilisées sans injection au cours des six derniers mois ${ }^{\mathrm{b}}$ (suite)

\begin{tabular}{|l|r|r|r|}
\hline Autres drogues $^{c}$ & 237 & 1751 & 13,5 \\
\hline Cannabis & 1698 & 2356 & 72,1 \\
\hline Alcool & 1472 & 2355 & 62,5 \\
\hline Crack & 1125 & 2352 & 47,8 \\
\hline Cocaïne & 1097 & 2354 & 46,6 \\
\hline Méthamphétamine & 1010 & 2349 & 43,0 \\
\hline Amphétamines & 836 & 2348 & 35,6 \\
\hline Méthadone & 824 & 2357 & 35,0 \\
\hline Benzodiazépines & 705 & 2349 & 30,0 \\
\hline Hydromorphone & 662 & 2351 & 28,2 \\
\hline Codéine & 645 & 2350 & 27,5 \\
\hline Morphine & 582 & 2354 & 24,7 \\
\hline Fentanyl & 462 & 2337 & 19,8 \\
\hline Héroïne & 462 & 2345 & 19,7 \\
\hline Oxycotin et oxycodone & 367 & 2347 & 15,6 \\
\hline Ecstasy & 223 & 2351 & 9,5 \\
\hline Champignons hallucinogènes & 214 & 2350 & 9,1 \\
\hline Talwin et Ritalin & 213 & 2352 & 9,1 \\
\hline Barbituriques & 200 & 2345 & 8,5 \\
\hline Autres drogues ${ }^{c}$ & 363 & 1809 & 20,1 \\
\hline Con $^{\prime}$ & & \\
\hline
\end{tabular}

Connaissance, accessibilité et utilisation d'une trousse contre les surdoses

Connaissance de l'existence des trousses contre les surdoses

Antécédents d'utilisation d'une trousse contre les surdoses

\begin{tabular}{|r|r|r|}
\hline 1276 & 1458 & 87,5 \\
\hline 408 & 1274 & 32,0 \\
\hline
\end{tabular}

Accessibilité des trousses contre les surdoses dans la collectivité des participants

\begin{tabular}{|l|r|r|r|}
\hline Oui & 1168 & 1276 & 91,5 \\
\hline Non & 44 & 1276 & 3,5 \\
\hline Ne sait pas & 64 & 1276 & 5,0 \\
\hline
\end{tabular}

Expériences de surdose

Antécédents de surdose au cours des six derniers mois ${ }^{\mathrm{e}}$

$374 \quad 1652$

Drogues ou substances utilisées lors de la dernière surdose $e^{b, d,}$

\begin{tabular}{|l|r|r|r|}
\hline Fentanyl & 128 & 298 & 43,0 \\
\hline Héroïne & 116 & 303 & 38,3 \\
\hline Méthamphétamine & 87 & 306 & 28,4 \\
\hline Cocaïne & 71 & 308 & 23,1 \\
\hline Alcool & 49 & 309 & 15,9 \\
\hline Cannabis & 40 & 307 & 13,0 \\
\hline Benzodiazépines & 35 & 305 & 11,5 \\
\hline
\end{tabular}


Tableau 5 : Consommation de drogues et antécédents de surdose des participants à l'enquête Track auprès des utilisateurs de drogues injectables au Canada, phase 4, 2017 à $2019(n=2$ 383) (suite)

\begin{tabular}{|c|c|c|c|}
\hline $\begin{array}{l}\text { Consommation de drogues et } \\
\text { antécédents de surdose }\end{array}$ & $\mathbf{n}$ & Total $^{a}$ & $\%$ \\
\hline \multicolumn{4}{|c|}{$\begin{array}{l}\text { Drogues ou substances utilisées consommées lors de la dernière } \\
\text { surdose }^{b, d, f} \text { (suite) }\end{array}$} \\
\hline Crack & 30 & 305 & 9,8 \\
\hline Morphine & 25 & 308 & 8,1 \\
\hline Méthadone & 23 & 308 & 7,5 \\
\hline Hydromorphone & 20 & 308 & 6,5 \\
\hline Autres drogues ${ }^{c}$ & 85 & 310 & 27,4 \\
\hline \multicolumn{4}{|c|}{$\begin{array}{l}\text { aLe total représente le nombre total de personnes associées à l'indicateur concerné, à l'exception } \\
\text { des personnes ayant répondu par " Je ne sais pas ", "Refus » et « Non indiqué » } \\
\text { b Les participants ont indiqué toutes les drogues (qu'ils avaient injectées, consommées ou } \\
\text { utilisées lors de la dernière surdose) à des fins non médicales au cours des six mois précédant } \\
\text { l'entrevue. Les drogues les plus fréquemment citées par les participants sont présentées. Les } \\
\text { réponses ne s'excluent pas mutuellement } \\
\text { "Les « autres drogues » comprennent les drogues associées à une fréquence inférieure à } 5 \% \\
\text { d Cette question n'a pas été posée dans les sites du réseau SurvUDI et dans le site de London } \\
\text { e Cette question n'a pas été posée dans les sites du réseau SurvUDI } \\
\text { †Parmi les participants qui ont souffert d'une surdose au cours des six derniers mois et qui ont } \\
\text { fourni une réponse }\end{array}$} \\
\hline
\end{tabular}

La majorité des participants de la phase 4 ont entendu parler des trousses contre les surdoses $(87,5 \%)$ et ont déclaré y avoir accès dans leur collectivité $(91,5 \%)$; une plus faible proportion d'entre eux avait déjà utilisé une trousse sur une autre personne (32\%). Près d'un quart $(22,6 \%)$ des participants ont souffert d'une surdose au cours des six mois précédant l'entrevue et les drogues les plus fréquemment utilisées lors de la dernière surdose étaient le fentanyl (43\%), I'héroïne (38,3\%), la méthamphétamine $(28,4 \%)$, la cocaïne $(23,1 \%)$ et l'alcool $(15,9 \%)$ (tableau 5$)$.

La drogue la plus fréquemment injectée pour l'ensemble des phases était la cocaïne (60\% à $81,6 \%$ ). Une tendance croissante de l'injection de l'hydromorphone (29,9\% à 50,1\%), de la méthamphétamine $(6,8 \%$ à $43,5 \%)$, du fentanyl $(1,7 \%$ à 24,3 $\%)$ et des amphétamines ( $7,9 \%$ à $21,5 \%)$ a été constatée entre la phase 1 et la phase 4. Sur l'ensemble des phases, la consommation de cannabis et d'alcool par d'autres voies que l'injection est restée élevée (tableau supplémentaire D).

\section{Comportements sexuels à risque}

Dans la phase 4, au moins une fois au cours des six mois précédant l'entrevue, parmi les participants ayant des antécédents de relations sexuelles, 35,2\% avaient eu deux partenaires sexuels ou plus, 59,2\% avaient utilisé le préservatif de manière irrégulière lors de relations vaginales ou anales avec un partenaire sexuel occasionnel, $84,9 \%$ avaient utilisé le préservatif de manière irrégulière lors de relations vaginales ou anales avec un partenaire sexuel régulier, et 15,7 \% avaient eu des relations sexuelles rémunérées (tableau 6). Parmi les participants qui avaient eu des relations sexuelles rémunérées, $30,7 \%$ n'avaient pas utilisé de préservatif lors de la dernière relation sexuelle rémunérée. La majorité des participants $(84,2 \%)$
Tableau 6 : Comportements sexuels à risque des participants à l'enquête Track auprès des utilisateurs de drogues injectables au Canada, phase 4, 2017 à 2019 $(\mathrm{n}=2,383)$

\begin{tabular}{|c|c|c|c|}
\hline Comportements sexuels à risque & $\mathbf{n}$ & Total $^{\mathrm{a}}$ & $\%$ \\
\hline $\begin{array}{l}\text { Deux partenaires sexuels ou plus au cours des } \\
\text { six derniers mois }{ }^{\mathrm{b}}\end{array}$ & 798 & 2270 & 35,2 \\
\hline $\begin{array}{l}\text { Utilisation irrégulière du préservatif lors } \\
\text { de relations vaginales ou anales avec un } \\
\text { partenaire sexuel occasionnel au cours des six } \\
\text { derniers mois }{ }^{c}\end{array}$ & 413 & 698 & 59,2 \\
\hline $\begin{array}{l}\text { Utilisation irrégulière du préservatif lors } \\
\text { de relations vaginales ou anales avec un } \\
\text { partenaire sexuel régulier au cours des six } \\
\text { derniers moisc }\end{array}$ & 1086 & 1279 & 84,9 \\
\hline $\begin{array}{l}\text { Relations sexuelles rémunérés au cours des } \\
\text { six derniers mois }\end{array}$ & 280 & 1786 & 15,7 \\
\hline $\begin{array}{l}\text { Absence de préservatif lors de la dernière } \\
\text { relation sexuelle rémunérée }\end{array}$ & 66 & 215 & 30,7 \\
\hline $\begin{array}{l}\text { Consommation de substances avant ou } \\
\text { pendant les relations sexuelles au cours des } \\
\text { six derniers mois }\end{array}$ & 1088 & 1292 & 84,2 \\
\hline $\begin{array}{l}\text { al représente le nombre total de personnes associées } \\
\text { ssonnes ayant répondu par « Je ne sais pas », « Refus, } \\
\text { nominateur exclut les participants sans antécédents d } \\
\text { ation irrégulière du préservatif est défini comme ne pp } \\
\text { atiti (c.-à-d. jamais, parfois ou souvent) lors des relatior } \\
\text { áe au site de London }\end{array}$ & 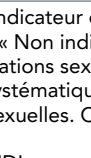 & "s & \\
\hline
\end{tabular}

ont déclaré consommer des substances avant ou pendant les relations sexuelles (tableau 6).

Sur l'ensemble des phases, parmi les participants ayant eu des antécédents de relations sexuelles au cours des six mois précédant l'entrevue, la proportion de ceux qui avaient eu deux partenaires sexuels ou plus et qui avaient eu des relations sexuelles rémunérées est restée relativement stable (tableau supplémentaire $D$ ).

\section{Prévalence du VIH et de l'hépatite $\mathrm{C}$ et connaissance du statut d'infection à ces maladies}

D'après les analyses en laboratoire, la prévalence du VIH était de $10,3 \%$. Parmi ces personnes séropositives pour le $\mathrm{VIH}$, $82,9 \%$ avaient connaissance de leur statut d'infection à la maladie (tableau 7). La proportion des participants chez qui la présence des anticorps anti-hépatite $C$ a été détectée était de $64,2 \%$, une mesure de l'exposition à vie à l'infection par le virus de l'hépatite $C$. De nombreux participants $(36,9 \%)$ étaient séropositifs pour l'ARN de l'hépatite $C$, un indicateur d'une infection actuelle à l'hépatite $C$, et, parmi ceux-ci, 50,1 \% avaient connaissance de leur statut de séropositivité pour l'ARN de I'hépatite $C$. Parmi les participants ayant fourni un échantillon biologique en quantité suffisante pour détecter la présence d'anticorps du VIH et d'ARN du VHC, 4,7 \% étaient séropositifs pour le VIH et séropositifs pour l'ARN du VHC; $4,3 \%$ étaient séropositifs pour le $\mathrm{VIH}$ et séronégatifs pour l'ARN du VHC; $32,3 \%$ étaient séronégatifs pour le $\mathrm{VIH}$ et séropositifs pour 
Tableau 7 : Prévalence et cascade de soins du VIH et de I'hépatite $C$ et connaissance du statut d'infection à ces maladies chez les participants à l'enquête Track auprès des utilisateurs de drogues injectables au Canada, phase 4, 2017 à $2019(n=2383)$ (suite)

\begin{tabular}{|c|c|c|c|}
\hline Prévalence du VIH et de I'hépatite C & $\mathbf{n}$ & Total ${ }^{\mathrm{a}}$ & $\%$ \\
\hline \multicolumn{4}{|c|}{ Prévalence du VIH et connaissance du statut d'infection à la maladie } \\
\hline Prévalence du VIH & 222 & 2162 & 10,3 \\
\hline $\begin{array}{l}\text { Connaissance du statut de séropositivité } \\
\text { pour le VIH }\end{array}$ & 179 & 216 & 82,9 \\
\hline
\end{tabular}

Cascade de soins du VIH (parmi les participants ayant connaissance de leur statut de séropositivité pour le VIH, $n=179$ )

\begin{tabular}{|l|r|r|r|}
\hline $\begin{array}{l}\text { Orientation vers les soins et les services liés } \\
\text { au VIH }\end{array}$ & 170 & 179 & 95,0 \\
\hline Antécédents de TA & 174 & 179 & 97,2 \\
\hline Suivi actuel d'un TA & 157 & 179 & 87,7 \\
\hline $\begin{array}{l}\text { Observance du TA, sans aucune dose omise } \\
\text { au cours du dernier mois }\end{array}$ & 34 & 80 & 42,5 \\
\hline $\begin{array}{l}\text { Autodéclaration d'une charge virale } \\
\text { indétectable pour le VIH' }\end{array}$ & 59 & 94 & 62,8 \\
\hline $\begin{array}{l}\text { Évitement des services liés au VIH en raison } \\
\text { d'antécédents de stigmatisation et de } \\
\text { discrimination au cours des douze derniers } \\
\text { mois }\end{array}$ & 43 & 95 & 45,3 \\
\hline
\end{tabular}

Prévalence de l'hépatite $C$ et connaissance du statut d'infection à la maladie

\begin{tabular}{|l|r|r|r|}
\hline Prévalence des anticorps anti-VHC c,h & 1375 & 2141 & 64,2 \\
\hline Prévalence de l'ARN du VHC c,i & 486 & 1316 & 36,9 \\
\hline $\begin{array}{l}\text { Connaissance du statut de séropositivité } \\
\text { pour l'ARN de l'hépatite Ci }\end{array}$ & 238 & 475 & 50,1 \\
\hline
\end{tabular}

Cascade de soins de l'hépatite $C$ (parmi les participants ayant

connaissance de leur statut de séropositivité pour l'ARN de l'hépatite $\mathrm{C}, \mathrm{n}=238)$

\begin{tabular}{|l|r|r|r|}
\hline Orientation vers les soins pour l'hépatite Ck & 115 & 237 & 48,5 \\
\hline $\begin{array}{l}\text { Antécédents de traitement contre l'hépatite } \\
C^{\prime}\end{array}$ & 25 & 236 & 10,6 \\
\hline $\begin{array}{l}\text { Suivi actuel d'un traitement contre l'hépatite } \\
\text { C }^{\prime}\end{array}$ & 9 & 236 & 3,8 \\
\hline
\end{tabular}

\section{Co-infection au VIH et à l'hépatite $\mathrm{C}^{\mathrm{m}}$}

\begin{tabular}{|l|r|r|r|}
\hline $\begin{array}{l}\text { Séropositivité pour le VIH et séropositivité } \\
\text { pour l'ARN de l'hépatite C }\end{array}$ & 62 & 1314 & 4,7 \\
\hline $\begin{array}{l}\text { Séropositivité pour le VIH et séronégativité } \\
\text { pour l'ARN de l'hépatite C }\end{array}$ & 57 & 1314 & 4,3 \\
\hline $\begin{array}{l}\text { Séronégativité pour le VIH et séropositivité } \\
\text { pour l'ARN de l'hépatite C }\end{array}$ & 424 & 1314 & 32,3 \\
\hline $\begin{array}{l}\text { Séronégativité pour le VIH et séronégativité } \\
\text { pour l'ARN de l'hépatite C }\end{array}$ & 771 & 1314 & 58,7 \\
\hline
\end{tabular}

Abréviations : ARN, acide ribonucléique; TA, traitement antirétroviral; VHC, virus de l'hépatite $C_{\text {; }}$ $\mathrm{VIH}$, virus de l'immunodéficience humaine

ae total représente le nombre total de personnes associées à l'indicateur concerné, à l'exception des personnes ayant répondu par " Je ne sais pas ", "Refus » et " Non indiqué »

b Parmi les participants ayant fourni un échantillon biologique en quantité suffisante pour détecter

Parmi les participants ayant fourni un échantillon biologique en quantite suffisante pour détecter présence du VIH

Les algorithmes de dépistage du VIH et de l'hépatite $\mathrm{C}$ sont présentés à l'annexe 2

d Parmi les participants chez qui la présence des anticorps anti-VIH a été détectée et qui ont déclaré leur diagnostic d'infection au VIH. Les participants ayant déclaré que les résultats de leur dernier test de dépistage du VIH étaient positifs et dont la séropositivité pour le VIH a été confirmée à la suite de l'analyse de l'échantillon biologique fourni au moment de l'entrevue ont été classés comme ayant connaissance de leur statut de séropositivité pour le VIH
Note de base (suite)

e Défini comme étant suivi par un médecin ou un fournisseur de soins de santé pour des services liés au VIH au moment de l'entrevue (au cours des six mois précédant l'entrevue dans le cas des sites du réseau SurvUDI et du site de London)

${ }^{\mathrm{f}}$ Cette question n'a pas été posée dans les sites du réseau SurvUDI $(n=65)$ et dans le site de London $(n=17)$. Le dénominateur exclut les participants pour lesquels il manque des données 9 Parmi les participants suivant un TA au moment de l'entrevue. Cette question n'a pas été posée dans les sites du réseau SurvUDI $(n=62)$. Le dénominateur exclut les participants pour lesquels il manque des données

${ }^{\mathrm{h}}$ Parmi les participants ayant fourni un échantillon biologique en quantité suffisante pour détecter la présence des anticorps anti-VHC

i Parmi les participants ayant fourni un échantillon biologique en quantité suffisante pour détecter Parmi les participants ayant fourni un échantillon biologique en quantite suffisante pour
la présence des anticorps anti-VHC et de l'ARN du VHC. Les analyses visant à détecter la présence de l'ARN du VHC n'ont pas été réalisées dans les sites du réseau SurvUDI ¡Parmi les participants chez qui la présence de I'ARN du VHC a été détectée et qui ont déclaré leur statut actuel de séropositivité pour l'hépatite $C$. Les participants ayant déclaré être actuellement infectés par l'hépatite $C$ et dont la séropositivité pour l'ARN de l'hépatite $C$ a été confirmée à la suite de l'analyse de l'échantillon biologique fourni au moment de l'entrevue ont été classés comme ayant conne l'éch l'ARN l'hépat été classés comme ayant connaissance de leur statut de séropositivite pour l'ARN de l'e $C$ "Défini comme étant suivi par un médecin ou un fournisseur de soins de santé pour des services liés à l'hépatite $C$ au moment de l'entrevue. Le dénominateur exclut les participants pour lesquels il manque des données

Le dénominateur exclut les participants pour lesquels il manque des données

m Parmi les participants ayant fourni un échantillon biologique en quantité suffisante pour détecter la présence des anticorps anti-VIH et de l'ARN du VHC. Les analyses visant à détecter la présence de l'ARN du VHC n'ont pas été réalisées dans les sites du réseau SurvUDI

I'ARN du VHC; et 58,7 \% étaient séronégatifs pour le $\mathrm{VIH}$ et séronégatifs pour l'ARN du VHC.

Au cours des quinze années qui se sont écoulées entre la phase 1 et la phase 4, la prévalence du VIH a diminué, passant de $14,9 \%$ à 10,3 \%. Parmi les participants séropositifs pour le $\mathrm{VIH}$, la proportion de ceux qui avaient connaissance de leur statut de séropositivité pour le VIH a légèrement augmenté (77,8 \% à 82,9\%). Sur l'ensemble des phases, la proportion des participants chez qui des anticorps anti-hépatite $C$ étaient détectés était relativement stable, soit environ les deux tiers des participants (64,2\% à $69 \%$ ) (tableau supplémentaire D).

\section{Cascade de soins du VIH et de l'hépatite C}

Les indicateurs permettant de mesurer la cascade de soins du VIH ont été examinés parmi les participants ayant connaissance de leur statut de séropositivité pour le VIH (tableau 7). La majorité d'entre eux étaient suivis par un médecin ou un fournisseur de soins de santé pour des services liés au VIH au moment de l'entrevue (95\%). La majorité d'entre eux avaient également des antécédents de traitement antirétroviral (TA) $(97,2 \%)$ et suivaient un TA au moment de l'entrevue $(87,7 \%)$. L'observance du TA, mesurée par la prise systématique des doses prescrites au cours du mois précédant l'entrevue, était de $42,5 \%$. Parmi les participants qui suivaient un TA au moment de l'entrevue, $62,8 \%$ ont déclaré une charge virale indétectable pour le $\mathrm{VIH}$. Près de la moitié des participants qui avaient connaissance de leur statut de séropositivité pour le VIH ont déclaré éviter les services liés au $\mathrm{VIH}$ en raison d'antécédents de stigmatisation et de discrimination au cours des douze derniers mois précédant l'entrevue (45,3\%).

Les indicateurs permettant de mesurer la cascade de soins de I'hépatite $C$ ont été examinés parmi les participants qui avaient connaissance de leur séropositivité pour l'hépatite $C$ (tableau 7). Près de la moitié $(48,5 \%)$ des participants ont déclaré avoir été orientés vers des soins pour l'hépatite $C_{\text {; }}$ une proportion plus faible $(10,6 \%)$ avait des antécédents de traitement contre I'hépatite C; une proportion encore plus faible (3,8\%) suivait actuellement un traitement contre I'hépatite $C$. 
Entre la phase 1 et la phase 4, parmi les participants ayant connaissance de leur statut de séropositivité pour le $\mathrm{VIH}$, l'orientation vers les soins et les services liés au $\mathrm{VIH}$ a augmenté $(88,1 \%$ à $95 \%)$, tout comme la proportion des participants suivant actuellement un TA (52 \% à 87,7\%). Sur l'ensemble des phases, seule environ la moitié des participants ayant connaissance de leur statut de séropositivité pour l'hépatite $C$ étaient suivis par un médecin pour leur infection à l'hépatite $C$, et la proportion de participants suivant actuellement un traitement contre l'hépatite $C$ était très faible (tableau supplémentaire $D$ ).

\section{Discussion}

Les utilisateurs de drogues injectables constituent un groupe associé à un risque d'infection important dans les épidémies de VIH et d'hépatite $C$ qui touchent le Canada (1). Les renseignements recueillis par l'enquête Track auprès des UDI au Canada permettent de contextualiser l'épidémiologie du $\mathrm{VIH}$, de l'hépatite $\mathrm{C}$ et les comportements à risque associés au sein de cette population; ils permettent également d'établir des comparaisons dans le temps et de générer de nouvelles données de référence sur les principaux indicateurs émergents, comme les antécédents de stigmatisation et de discrimination, les surdoses et le recours à la PrEP. Les facteurs associés à un risque accru d'infection au VIH et à l'hépatite $C$ dans les enquêtes précédentes ont également été mis en évidence au sein de cet échantillon d'UDI. Les marqueurs de la pauvreté et de la marginalisation, illustrés notamment par le nombre élevé de personnes se trouvant dans une situation de logement précaire et/ou ayant des antécédents d'incarcération, étaient fréquents. La majorité des participants ont également fait état d'antécédents de stigmatisation et de discrimination, ainsi que de violences physiques, sexuelles et/ou psychologiques durant l'enfance ou de la part d'un partenaire sexuel.

Des taux élevés de dépistage du VIH et de l'hépatite $\mathrm{C}$ ainsi que le recours aux programmes de distribution d'aiguilles et de seringues ont été constatés. Néanmoins, le recours aux autres services essentiels de réduction des méfaits était plus faible. Moins de la moitié des participants ont déclaré avoir recouru à un traitement de substitution aux opiacés ou fréquenté un site d'injection ou de consommation supervisées au cours de l'année précédente. Les renseignements obtenus au cours de la phase 4 concernant la consommation de drogues et les comportements d'injection ont montré qu'une large proportion de participants empruntaient des aiguilles ou des seringues et d'autres matériels d'injection usagés. La majorité des participants $(59,2 \%)$ ont déclaré une utilisation irrégulière du préservatif avec un partenaire sexuel occasionnel et $84,2 \%$ ont déclaré une consommation de substances avant ou pendant les rapports sexuels, ces deux facteurs étant associés à la transmission des ITSS, y compris la syphilis. Peu de participants (14,3\%) avaient connaissance de la prophylaxie préexposition, et seulement $0,3 \%$ des participants n'ayant pas déclaré de diagnostic d'infection au VIH avaient recours à la PrEP.
Les résultats de la surveillance nationale concordent en grande partie avec ceux des autres systèmes de surveillance biocomportementale intégrée ayant des épidémies de $\mathrm{VIH}$ et d'hépatite $C$ comparables. Plus particulièrement, parmi les UDI interrogés aux États-Unis, en Australie et au Royaume-Uni, les niveaux des indicateurs de prévention et de dépistage (analyses visant à détecter la présence du VIH et de l'hépatite $C$, recours à un traitement de substitution aux opiacés), les comportements d'injection (emprunt d'aiguilles ou de seringues usagées, emprunt d'autres matériels d'injection usagés) et les pratiques sexuelles (relations sexuelles rémunérées, relations non protégées) étaient similaires (6-8). Les précédentes enquêtes régionales auprès des UDI au Canada ont mis en évidence des niveaux de précarité similaires en matière de logement $(9,10)$, et constaté des antécédents de violence $(10)$ et d'abus $(9,10)$ chez une large proportion de participants.

La population des UDI au Canada a été particulièrement touchée par la crise actuelle des opiö̈des et les autres décès par surdose de drogue. La consommation accrue de méthamphétamine, de fentanyl et d'analgésiques opioïdes observée chez les participants de la phase 4 fait écho à cette tendance alarmante. Les résultats de surveillance de la phase 4 ont permis de recueillir des renseignements sur les nouveaux indicateurs de surdose. Bien que la connaissance et l'accessibilité des trousses contre les surdoses étaient élevées, $22,6 \%$ des participants avaient souffert d'une surdose au cours des six mois précédant l'entrevue, et le fentanyl et l'héroïne étaient les drogues les plus fréquemment consommées lors de la dernière surdose.

Alors que la prévalence du VIH chez les participants de la phase $4(10,3 \%)$ avait diminué par rapport à la phase 1 (réalisée entre 2003 et 2005), elle restait malgré tout élevée, avec des taux presque dix fois supérieurs à ceux enregistrés chez les UDI en Australie et au Royaume-Uni $(7,8)$. Une proportion légèrement plus élevée avait connaissance de leur statut de positivité pour le $\mathrm{VIH}$ dans la phase 4 (82,9\%) par rapport aux phases précédentes. Pour la première fois, la prévalence de l'ARN de I'hépatite $\mathrm{C}$ a été mesurée dans l'enquête Track auprès des UDI et celle-ci s'est révélée élevée (36,9\%). En outre, seuls $50,1 \%$ des participants avaient connaissance de leur statut de séropositivité pour l'ARN de l'hépatite C (infection actuelle à l'hépatite C).

Presque tous les participants ayant connaissance de leur statut de positivité pour le VIH ont été orientés vers les soins et les services liés au VIH et suivaient actuellement un TA. Toutefois, moins des deux tiers $(62,3 \%)$ ont déclaré une charge virale indétectable et $45,3 \%$ ont déclaré éviter les services liés au VIH en raison d'antécédents de stigmatisation et de discrimination. Des taux beaucoup plus faibles pour ce qui est de l'orientation vers les soins $(48,5 \%)$ et du suivi actuel d'un traitement $(3,8 \%)$ ont été observés chez les participants ayant autodéclaré une infection actuelle à l'hépatite $C$. Un faible nombre d'UDI orientés vers les soins et le traitement pour l'hépatite a été enregistré dans les autres enquêtes régionales au Canada (11). 
Les résultats de l'enquête Phase 4 Tracks auprès des UDI peuvent éclairer les stratégies fondées sur des preuves pour combler les lacunes dans les approches de prévention, de dépistage et de lien avec les soins. Cela peut inclure un meilleur lien avec la thérapie de substitution aux opioïdes et les sites d'injection ou de consommation supervisés, et améliorer l'accès aux services de santé et aux services sociaux pour la santé mentale et les toxicomanies (12). La confluence de taux élevés d'hépatite $C$ combinée à une mauvaise sensibilisation, à un partage continu, mais réduit des aiguilles et à une utilisation irrégulière du préservatif, malgré l'augmentation des taux d'adoption du programme, souligne la nécessité de programmes de réduction des méfaits pour continuer à évoluer afin de relever ces défis.

\section{Forces et faiblesses}

L'enquête Track auprès des UDI offre une mine de renseignements sur le $\mathrm{VIH}$ et l'hépatite $\mathrm{C}$ chez les UDI de plusieurs sites répartis à travers du Canada, et permet de cerner les tendances des principaux indicateurs depuis 2003. Elle constitue d'ailleurs l'unique source nationale de renseignements de la sorte, et a été utilisée à l'échelon local, provincial et fédéral pour éclairer et orienter les mesures de santé publique adoptées à l'égard de cette population. II convient cependant de noter que l'enquête Track repose sur l'échantillonnage non probabiliste; il se peut ainsi que les résultats ne soient pas représentatifs de tous les UDI d'un site donné ou de l'ensemble du Canada. À l'exception des résultats de laboratoire, ces résultats sont fondés sur des questionnaires administrés par un intervieweur et sur des données autodéclarées; il est donc possible que certains comportements à risque aient été surreprésentés ou sous-représentés.

\section{Conclusion}

L'enquête a mis en évidence des niveaux élevés de précarité en matière de logement, des antécédents de stigmatisation et de discrimination, l'emprunt de matériel d'injection usagé et I'utilisation irrégulière du préservatif. La prévalence du VIH et de I'ARN de I'hépatite $C$ était élevée chez les UDI dans certaines régions du Canada. D'importantes lacunes liées à l'orientation vers les soins et le traitement de l'hépatite $C$ ont été relevées. Ces résultats soulignent la nécessité d'assurer un accès continu aux services de dépistage et de prévention, de mettre en place des stratégies ciblées pour éliminer les barrières à l'accès aux soins et au traitement du VIH et de l'hépatite $\mathrm{C}$, et d'améliorer de manière permanente les efforts à l'égard du logement précaire, de la santé mentale et de la toxicomanie.

\section{Déclaration des auteurs}

J. T. - Conceptualisation, analyse formelle, méthodologie, administration de projet, rédaction (version initiale, révision et édition)

J. Z. - Conceptualisation, organisation des données, analyse formelle, rédaction (version initiale, révision et édition)

A. L. - Conceptualisation, analyse formelle, rédaction (révision et édition)
F. C. - Conceptualisation, méthodologie, rédaction (révision et édition)

M. B. - Conceptualisation, méthodologie, administration de projet, rédaction (révision et édition)

D. P. - Conceptualisation, acquisition du financement, méthodologie, rédaction (révision et édition)

\section{Conflit d'intérêts}

Aucun.

\section{Remerciements}

La mise en place du système de surveillance Track a été rendue possible grâce à une collaboration fructueuse entre l'Agence de la santé publique du Canada (y compris le Laboratoire national de microbiologie) et les autorités de santé, les partenaires et les organismes communautaires provinciaux, régionaux et locaux. Les auteurs tiennent à remercier chaleureusement pour leur contribution les participants, les équipes des sites sentinelles (y compris les coordinateurs et les intervieweurs de l'enquête) et les investigateurs principaux de chaque site : M. Alary; K. Chokani; J. DeMille; B. Enns; M. Gully; B. Hanley; M. Hennink; P. Leclerc; C. Mackie; S. Marshall; C. Morissette; É. Roy; S. Shaw; C. Smith; A. Vanderlaan; et $D$. Warren. Les auteurs tiennent également à remercier $\mathrm{C}$. Archibald pour ses conseils avant et pendant la collecte des données et pour son examen du manuscrit, ainsi que C. Daniuk pour son aide dans l'analyse des échantillons de sang séché.

\section{Financement}

L'enquête Track auprès des utilisateurs de drogues injectables au Canada est financée par l'Agence de la santé publique du Canada, et bénéficie de contributions en nature de la part des autorités de santé et des organismes communautaires régionaux et locaux.

\section{Références}

1. Agence de la santé publique du Canada. Résumé: Estimations de l'incidence et de la prévalence du $\mathrm{VIH}$, et des progrès réalisés par le Canada en ce qui concerne les cibles 90-90-90 pour le VIH, 2016. Gouvernement du Canada; 2018. https:// www.canada.ca/content/dam/phac-aspc/documents/services/ publications/diseases-conditions/summary-estimates-hiv-incidenc e-prevalence-canadas-progress-90-90-90/pub-fra.pdf

2. Degenhardt L, Charlson F, Stanaway J, Larney S, Alexander LT, Hickman M, Cowie B, Hall WD, Strang J, Whiteford H, Vos T. Estimating the burden of disease attributable to injecting drug use as a risk factor for HIV, hepatitis $C$, and hepatitis B: findings from the Global Burden of Disease Study 2013. Lancet Infect Dis 2016;16(12):1385-98. DOI PubMed

3. Agence de la santé publique du Canada. I-Track: surveillance améliorée du VIH et de l'hépatite $\mathrm{C}$ ainsi que des comportements à risque chez les utilisateurs de drogues injectables au Canada Rapport sur la phase 3 (2010-2012). Gouvernment du Canada; 2018. https://www.canada.ca/en/public-health/services/publications/ diseases-conditions/itrack-enhanced-surveillance-hiv-hepatitisassociated-risk-behaviours-people-who-inject-drugs-canada-phase-3. html 
4. Organisation mondiale de la Santé /IRIS. Directives pour la surveillance des populations les plus exposées au VIH. Genève : OMS; 2011. https://apps.who.int/iris/bitstream/hand le/10665/44668/9789242501667_fre.pdf?sequence=1\&isAllowed=y

5. Leclerc P, Roy É, Morissette C, Alary M, Parent R, Blouin K. Surveillance des maladies infectieuses chez les utilisateurs de drogues par injection - Épidémiologie du VIH de 1995 à 2014 - Épidémiologie du VHC de 2003 à 2016. Institut national de santé publique du Québec; 2018. https://www.inspq.qc.ca/sites/ default/files/publications/2400_surveillance_maladies_infectieuses_ utilisateurs_drogue_injection.pdf

6. Centers for Disease Control and Prevention. HIV Infection, Risk, Prevention, and Testing Behaviors among Persons Who Inject Drugs-National HIV Behavioral Surveillance: Injection Drug Use, 20 U.S. Cities, 2015. Atlanta (GA); CDC: 2018. https://www.cdc.gov/hiv/ pdf/library/reports/surveillance/cdc-hiv-hssr-nhbs-pwid-2015.pdf

7. Heard S, Iversen J, Geddes L, Maher L. Australian Needle Syringe Program Survey National Data Report 2014-2018: Prevalence of HIV, HCV and injecting and sexual behaviour among NSP attendees. Sydney (NSW): Kirby Institute, UNSW Sydney; 2019. https://kirby. unsw.edu.au/sites/default/files/kirby/report/ANSPS_National-DataReport-2014-2018.pdf

8. Public Health England. Unlinked Anonymous Monitoring (UAM) Survey of HIV and viral hepatitis among PWID: 2019 report. London (UK): PHE; 2019. https://assets.publishing.service.gov.uk/ government/uploads/system/uploads/attachment_data/file/825117/ hpr2919_UAM-PWID.pdf

\section{Annexes}

Annexe 1 : Enquête Track auprès des utilisateurs de drogues injectables au Canada - nombre total de participants et participation des sites sentinelles, phases 1 à 4

Annexe 2 : Algorithmes de dépistage du VIH et de I'hépatite C
9. Miller CL, Pearce ME, Moniruzzaman A, Thomas V, Christian CW, Schechter MT, Spittal PM. The Cedar Project: risk factors for transition to injection drug use among young, urban Aboriginal people. CMAJ. July 12, 2011;183(10):1147-54. DOI

10. Dong H, Hayashi K, Singer J, Milloy MJ, DeBeck K, Wood E, Kerr T. Trajectories of injection drug use among people who use drugs in Vancouver, Canada, 1996-2017: growth mixture modeling using data from prospective cohort studies. Addiction 2019;114(12):2173-86. DOI PubMed

11. Socías ME, Ti L, Wood E, Nosova E, Hull M, Hayashi K, Debeck K, Milloy MJ. Disparities in uptake of direct-acting antiviral therapy for hepatitis $C$ among people who inject drugs in a Canadian setting. Liver Int 2019;39(8):1400-7. DOI PubMed

12. Agence de la santé publique du Canada. Un Cadre d'action Pancanadien sur les ITSS : Réduction des répercussions sur la santé des infections transmissibles sexuellement et par le sang au Canada d'ici 2030. Gouvernement du Canada; 2018. https://www.canada. $\mathrm{ca} /$ content/dam/phac-aspc/documents/services/infectious-diseases/ sexual-health-sexually-transmitted-infections/reports-publications/ sexually-transmitted-blood-borne-infections-action-framework/ infections-transmissibles-sexuellement-sang-cadre-action.pdf

\section{Tableaux supplémentaires}

Tableau A : Indicateurs de prévention et de dépistage par caractéristiques sociodémographiques et déterminants sociaux de la santé des participants à l'enquête Track auprès des utilisateurs de drogues injectables au Canada, phase 4, 2017 à $2019(n=2383)$

Tableau B : Indicateurs de comportements d'injection et de consommation de drogue par caractéristiques sociodémographiques et déterminants sociaux de la santé des participants à l'enquête Track auprès des utilisateurs de drogues injectables au Canada, phase 4, 2017 à $2019(n=2$ 383)

Tableau C : Indicateurs de comportements sexuels à risque par caractéristiques sociodémographiques et déterminants sociaux de la santé des participants à l'enquête Track auprès des utilisateurs de drogues injectables au Canada, phase 4, 2017 à $2019(n=2383)$

Tableau D : Indicateurs sélectionnés selon la phase de l'enquête Track auprès des utilisateurs de drogues injectables au Canada, phases 1 à 4, 2003 à 2019 
Annexe 1 : Enquête Track auprès des utilisateurs de drogues injectables au Canada - nombre total de participants et participation des sites sentinelles, phases 1 à 4

\begin{tabular}{|c|c|c|c|c|}
\hline Détails de la phase & $\begin{array}{c}\text { Phase } 1 \\
2003 \text { à } 2005\end{array}$ & $\begin{array}{c}\text { Phase } 2 \\
2005 \text { à } 2008\end{array}$ & $\begin{array}{c}\text { Phase } 3 \\
2010 \text { à } 2012\end{array}$ & $\begin{array}{c}\text { Phase } 4 \\
2017 \text { à } 2019\end{array}$ \\
\hline Nombre total de participants & 2986 & 2982 & 2687 & 2383 \\
\hline Nombre de sites sentinelles & 7 & 10 & 11 & 14 \\
\hline \multicolumn{5}{|l|}{ Site sentinelle } \\
\hline Whitehorse (Yn) & - & - & 55 & 49 \\
\hline Centre et nord de l'île de Vancouver (C.-B.) & - & 220 & - & 179 \\
\hline Victoria (C.-B.) & 253 & 249 & - & - \\
\hline Prince George (C.-B.) & - & 156 & 150 & - \\
\hline Edmonton (Alb.) & 272 & 248 & 183 & - \\
\hline Prince Albert (Sask.) & - & - & - & 184 \\
\hline Regina (Sask.) & 250 & 250 & 251 & 205 \\
\hline Winnipeg (Man.) & 245 & - & - & 181 \\
\hline Thunder Bay (Ont.) & - & 149 & 138 & 200 \\
\hline Sudbury (Ont.) & 150 & 147 & 148 & - \\
\hline London (Ont.) & - & - & 204 & 206 \\
\hline Hamilton (Ont.) & - & - & - & 157 \\
\hline Toronto (Ont.) & 257 & 255 & 260 & - \\
\hline Kingston (Ont.) & - & 224 & 200 & - \\
\hline Réseau SurvUDI (Qc) & 1559 & 1084 & 937 & $692^{b}$ \\
\hline Nouveau-Brunswick & - & - & - & 200 \\
\hline Halifax (N.-É.) & - & - & 161 & - \\
\hline Terre-Neuve & - & - & - & 130 \\
\hline
\end{tabular}




\title{
Annexe 2 : Algorithmes de dépistage du VIH et de l'hépatite C
}

\begin{abstract}
Algorithmes de dépistage du VIH
Pour les sites extérieurs au réseau SurvUDI, le statut de positivité pour le VIH a dans un premier temps été déterminé en analysant les échantillons de sang séché au moyen de l'essai antigène-anticorps anti-VIH Bio-Rad GS, suivi de tests de confirmation réalisés à l'aide de l'essai quantitatif Roche COBAS AmpliPrep/COBAS Taqman VIH-1 v2.0 (London) ou de l'essai qualitatif Roche COBAS AmpliPrep/COBAS Taqman VIH-1 v2.0 (Nouveau-Brunswick, Terre-Neuve et Regina). Pour les autres sites extérieurs au réseau SurvUDI (île de Vancouver, Thunder Bay, Whitehorse, Winnipeg, Prince Albert et Hamilton), compte tenu de la récurrence des échantillons de faible volume, le statut de séropositivité pour le $\mathrm{VIH}$ a été déterminé par des tests de dépistage et des tests de confirmation basés sur deux essais immunoenzymatiques (EIA) distincts. Ainsi, dans la plupart des cas, le volume des échantillons était suffisant pour vérifier la présence du $\mathrm{VIH}$ et de l'hépatite $\mathrm{C}$. II est peu probable que le changement apporté aux algorithmes ait pu influer sur les résultats. Les algorithmes sont décrits de manière plus détaillée ci-dessous.
\end{abstract}

London : Le dépistage du VIH a été réalisé au moyen de l'essai antigène-anticorps anti-VIH Bio-Rad GS. Un résultat non réactif témoignait d'une absence d'infection au $\mathrm{VIH}$. Des tests de confirmation ont été réalisés sur les résultats de dépistage réactifs au moyen de l'essai quantitatif Roche COBAS AmpliPrep/COBAS Taqman VIH-1 v2.0. Un résultat détecté témoignait d'une infection au $\mathrm{VIH}$. Lorsque l'essai antigène-anticorps anti-VIH Bio-Rad GS était positif, et que le résultat de l'essai VIH-1 Roche COBAS ApliPrep/ COBAS Taqman v2.0 n'était pas détecté, un second EIA (système Microelisa AVIOQ VIH-1) était réalisé. Un résultat réactif à l'essai antigène-anticorps anti-VIH Bio-Rad GS et au système Microelisa AVIOQ VIH-1 témoignait d'une infection au VIH.

Nouveau-Brunswick, Terre-Neuve et Regina : Le dépistage du VIH a été réalisé au moyen de l'essai antigène-anticorps anti-VIH Bio-Rad GS (essai Bio-Rad). Un résultat non réactif témoignait d'une absence d'infection au VIH. Des tests de confirmation ont été réalisés sur les résultats d'analyse réactifs au moyen de l'essai qualitatif Roche COBAS AmpliPrep/COBAS Taqman VIH-1 v2.0 (essai Roche). Un résultat détecté témoignait d'une infection au VIH. Lorsque l'essai Bio-Rad était réactif, et que le résultat de l'essai Roche n'était pas détecté, un second EIA, le système Microelisa AVIOQ VIH-1 (essai Avioq) était réalisé pour apporter une confirmation définitive. Un résultat réactif à l'essai Bio-Rad et à l'essai Avioq témoignait d'une infection au VIH. Un résultat réactif à l'essai Bio-Rad, un résultat non détecté à l'essai Roche et un résultat non réactif ou indéterminé (c'est-à-dire un résultat d'absorbance proche de la valeur limite associée à un résultat réactif ou non réactif, sans toutefois être identique à celle-ci) à l'essai Avioq étaient globalement interprétés comme un résultat indéterminé.

Île de Vancouver, Thunder Bay, Whitehorse, Winnipeg, Prince Albert et Hamilton : Le dépistage du VIH a été réalisé au moyen de I'essai antigène-anticorps anti-VIH Bio-Rad GS (essai Bio-Rad). Un résultat non réactif témoignait d'une absence d'infection au VIH. Des tests de confirmation ont été réalisés sur les résultats de dépistage réactifs au moyen d'un second EIA, le système Microelisa AVIOO VIH-1 (essai Avioq). Un résultat réactif témoignait d'une infection au VIH. Lorsque l'essai Bio-Rad était réactif ou que l'essai Avioq était non réactif ou indéterminé (c.-à-d. avec un résultat d'absorbance proche de la valeur limite associée à un résultat réactif ou non réactif, sans toutefois être identique à celle-ci), l'essai qualitatif VIH-1 Roche COBAS AmpliPrep/COBAS Taqman v2.0 (essai Roche) était réalisé pour apporter une confirmation définitive. Un résultat réactif à l'essai Bio-Rad et un résultat détecté à l'essai Roche témoignaient d'une infection au $\mathrm{VIH}$. Un résultat réactif à l'essai Bio-Rad, un résultat non réactif ou indéterminé à l'essai Avioq et un résultat non détecté à l'essai Roche étaient globalement interprétés comme un résultat indéterminé.

Pour les sites du réseau SurvUDI, les échantillons de salive ont été soumis à des analyses visant à détecter la présence du VIH au Laboratoire de santé publique du Québec et à l'Institut national de santé publique du Québec; ces analyses ont été réalisées au moyen de l'EIA Bio-Rad GS PLUS O VIH-1/VIH-2, un essai diagnostique approuvé par Santé Canada et validé dans l'étude SurvUDI pour les échantillons de salive. Des tests de confirmation n'ont pas été réalisés sur les échantillons associés à plusieurs reprises à un résultat réactif. Un résultat positif témoignait d'une infection au $\mathrm{VIH}$.

\section{Algorithmes de dépistage de l'hépatite C}

Pour les sites extérieurs au réseau SurvUDI, le dépistage de l'hépatite $C$ a été réalisé au moyen de l'EIA Ortho ${ }^{\circledR}$ VHC version 3.0 (essai Ortho). Un résultat non réactif témoignait d'une absence d'antécédents d'infection à l'hépatite $C$. Un résultat réactif témoignait d'une exposition à vie à l'hépatite $C$. Des tests de confirmation ont été réalisés sur les résultats de dépistage réactifs et indéterminés (c.-à-d. les résultats d'absorbance proches de la valeur limite associée à un résultat réactif ou non réactif, sans toutefois être identique à celle-ci) au moyen de l'essai quantitatif Roche COBAS AmpliPrep/COBAS Taqman VHC v2.0 (essai Roche). Un résultat détecté 


\section{Annexe 2 : Algorithmes de dépistage du VIH et de l'hépatite $\mathrm{C}$ (suite)}

témoignait d'une infection actuelle à l'hépatite $C$ et un résultat non détecté témoignait d'une exposition à vie à l'hépatite $C$. Pour les échantillons associés à un résultat indéterminé à l'essai Ortho, un résultat détecté à l'essai Roche témoignait d'une infection actuelle à l'hépatite $C$ et un résultat non détecté à l'essai Roche était interprété comme un résultat indéterminé.

Pour les sites du réseau SurvUDI, le dépistage des anticorps anti-hépatite $C$ dans les échantillons de salive a été réalisé au moyen de I'EIA Ortho ${ }^{\circledR}$ hépatite $C$ version 3.0 par les laboratoires de l'Institut national de santé publique du Québec. Les tests de confirmation n'ont pas été réalisés sur les échantillons associés à plusieurs reprises à un résultat positif. Un résultat positif témoignait d'une infection antérieure ou actuelle à l'hépatite $C$, sans faire la distinction entre une infection aiguë, chronique ou passée. La validation de ce test en vue d'une utilisation avec les échantillons de salive a été effectuée dans l'étude SurvUDI.

\section{Sensibilité et spécificité des essais de laboratoire}

D'après la documentation du fabricant et les données de validation interne, la spécificité de l'EIA antigène-anticorps anti-VIH Bio-Rad GS, du système Microelisa Avioq VIH-1 et de l'essai qualitatif Roche COBAS AmpliPrep/COBAS TaqMan VIH-1 v2.0 est égale ou supérieure à 99,9\% sur les échantillons de sang séché. De même, chaque essai affiche une sensibilité de $100 \%$, à l'exception de l'EIA antigène-anticorps anti-VIH Bio-Rad GS qui présente une sensibilité de 96,6\%. Le seuil de quantification de l'essai quantitatif Roche COBAS/AmpliPrep TaqMan VIH-1 v2.0 sur les échantillons de sang séché est de $616 \mathrm{copies} / \mathrm{ml}$.

D'après les données de validation interne, le système d'essai ORTHO ELISA VHC v3.0 présente une spécificité et une sensibilité de 100 \%. Le seuil de quantification de l'essai Roche COBAS AmpliPrep/COBAS TaqMan VHC v2.0 est de 355 IU/ml. 